\section{Biological Control and Microbial Ecology Draft Genome Sequence Data of Glutamicibacter sp. FBE-19, a Bacterium Antagonistic to the Plant Pathogen Erwinia tracheiphila}

\author{
Benzhong Fu, ${ }^{1,2}$ Olakunle Olawole, ${ }^{1}$ and Gwyn A. Beattie ${ }^{1, \dagger}$ \\ ${ }^{1}$ Department of Plant Pathology \& Microbiology, lowa State University, Ames, IA 50011, U.S.A. \\ ${ }^{2}$ Hubei Key Laboratory of Quality Control of Characteristic Fruits and Vegetables, College of Life Science \\ and Technology, Hubei Engineering University, Xiaogan, Hubei 432000, China
}

\begin{abstract}
Glutamicibacter sp. FBE-19 was isolated based on its strong antagonism to the cucurbit bacterial blight pathogen Erwinia tracheiphila on plates. Members of the Glutamicibactergenus can promote plant growth under saline conditions and antagonize fungi on plates via chitinolytic activity; however, their production of antibacterial compounds has not been examined. Here, we report the genome sequence of strain FBE-19. The genome is $3.85 \mathrm{Mbp}$ with a $\mathrm{G}+\mathrm{C}$ content of $60.1 \%$ and comprises 3,791 genes. Genes that may contribute to its antagonistic activity include genes for the secondary metabolites stenothricin, salinosporamide $A$, a second $\beta$-lactone compound, and a carotenoid. The Glutamicibacter sp. FBE-19 genome data may be a useful resource if this strain proves to be an effective biocontrol agent against $E$. tracheiphila.
\end{abstract}

\section{Genome Announcement}

Cucurbit bacterial wilt caused by Erwinia tracheiphila is an economically important disease in the Midwestern and Northeastern United States that is primarily controlled by managing the insects that transmit it, namely striped and spotted cucumber beetles (Rojas et al. 2015). Biological control of this pathogen would provide an alternative management approach, and one that would be desirable for integration into organic production systems. Thus far, there are few reports evaluating potential agents for biocontrol of this pathogen (Roberts et al. 2018).

In working with $E$. tracheiphila in the laboratory, a serendipitous contaminant was discovered that induced a large zone of inhibition on a lawn of the E. tracheiphila strain BHKY grown on King's B agar (Fig. 1). This zone of inhibition was larger than those produced by other bacterial strains, including Paraburkholderia phytofirmans (PSJN-1) and Burkholderia pyrocinia (55B1 and FP62), that had been examined as potential biocontrol agents (unpublished data). We designated this antagonistic strain FBE-19. Strain FBE-19 produced a similar-sized zone of inhibition in both the presence and absence of exogenous $\mathrm{FeCl}_{3}(10 \mu \mathrm{M})$, indicating that the zone was not due to iron competition. We found that strain FBE-19 was highly antagonistic to all of the 42 E. tracheiphila strains examined, which included strains representing two distinct clades of E. tracheiphila (Shapiro et al. 2018), as well as to the closely related enteric bacterial species Pantoea stewartii (unpublished data).

Strain FBE-19 was identified by 16 S ribosomal RNA (rRNA) sequencing as Glutamicibacter sp. This genus currently has six named species, and a BLASTn search of the NCBI database indicated that FBE-19 is most closely related to Glutamicibacter halophytocola strains DR408 and KLBMP-5180 (98\% identity with each); however, when the 16S rRNA sequences of several

${ }^{\dagger}$ Corresponding author: G. A. Beattie; gbeattie@ iastate.edu

The author(s) declare no conflict of interest.

Accepted for publication 5 November 2020.

\section{Keywords}

antagonistic, bacterial pathogens, biological control, bioinformatics,

disease control and pest management, Erwinia tracheiphila, genome resource, genomics, Glutamicibacter sp. 


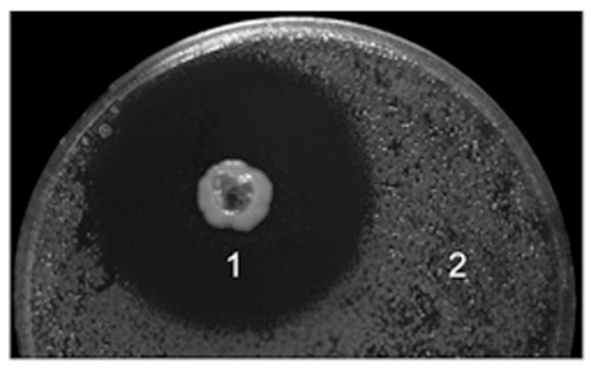

B

Tree scale: $0.01 \longmapsto$ G.creatinolyticus LGCM259

G.arilaitensis Re117

G.nicotianae OTC-16

G.mishrai S5-52

FBE-19

G.halophytocola KLBMP5180

Fig. 1. Antagonism and phylogeny of Glutamicibacter sp. strain FBE-19. A, Antagonistic effect of (1) Glutamicibacter sp. FBE-19 against Erwinia tracheiphila strain BHKY on King's B agar as compared with (2) a water control. B, Phylogenetic tree of selected Glutamicibacter strains based on ribosomal RNA proteins (constructed by Mauve2.4.0 and displayed by iTOL).

Glutamicibacter spp. were used for alignment, a phylogenetic tree analysis using MEGA7 indicated insufficient resolution to determine the Glutamicibacter sp.

The genus Glutamicibacter was reclassified in 2016 from Arthrobacter (Busse 2016). Bacteria of the genus Glutamicibacter have been found in a variety of environments, including from animals (Santos et al. 2020), cheese (Irlinger et al. 2005), and plants (Qin et al. 2018). Glutamicibacter isolates were shown to enhance the growth of the halophytic plant Limonium sinense, as well as tomato, rice, and soybean plants under saline or drought conditions (Feng et al. 2017; Ji et al. 2020; Nishu et al. 2019; Qin et al. 2018; Xiong et al. 2019). Although G. nicotianae was recently evaluated as an antagonist of quorum signal-mediated virulence of a soft rot pathogen (Vesuna and Nerurkar 2020), Glutamicibacter strains have not yet been examined for biocontrol activity mediated by the production of antimicrobial compounds. The genome sequence of Glutamicibacter sp. FBE-19 reported here may provide insights into traits contributing to the antagonism of $E$. tracheiphila or, more generally, to impacts of this genus on plant health.

Glutamicibacter sp. FBE-19 was grown on King's B medium for 2 days at $28^{\circ} \mathrm{C}$ and harvested for DNA extraction using the Gentra Puregene bacterial DNA extraction kit (Qiagen). The genomic DNA quality was assessed by visualization on a gel and was quantified using a Qubit Fluorometer. High-quality DNA (200 ng) was sequenced using the HiSeq3000 platform at the DNA Facility at lowa State University, generating 100-bp paired-end reads. In total, $6,980,054$ reads were obtained, and no sequences were flagged as poor quality when using FastQC (v0.11.9) (Andrews 2010) for quality analysis. The raw reads were trimmed with Trimmomatic (v0.39) using the parameters ILLUMINACLIP: Nexte, PE adapter:2:30:10:2: keep Both Reads, LEADING:3, TRAILING:3, SLIDINGWINDOW:4:15, HEADCROP:14, and MINLEN:36. In total, 6,583,430 reads were used for the subsequent assembly. The genome was assembled by SPAdes v3.14.0 (Bankevich et al. 2012). The quality of the assembly was evaluated by QUAST v5.0.2 (Mikheenko et al. 2018). The genome was assembled into 22 contigs (>1,000 bp), with the largest contig being $798,790 \mathrm{bp}$ and a total length of $3,846,453 \mathrm{bp}$. The $N_{50}$ was 334,508 and $L_{50}$ was 4 . The $G+C$ content of the genome is $60.1 \%$.

The gene starts were predicted using the GeneMarkS program (version 4.17) (Besemer et al. 2001), which predicted a total of 3,665 genes. Of these, 1,833 were assigned to 23 Kyoto Encyclopedia of Genes and Genomes orthology groups using BlastKOALA (Kanehisa et al. 2016). The genome was annotated by RASTtk (Brettin et al. 2015) (Table 1), which predicted a total of 3,791 genes, with 1,903 on the positive strand and 1,888 on the negative strand, and the coding sequences composing $90.0 \%$ of the genome. The annotated genes included 3,704 protein-coding sequences (CDSs), with 2,487 (67\%) with some functional prediction and 1,217 $(34 \%)$ annotated as hypothetical proteins. The average CDS size was $929 \mathrm{bp}$, which varied from $114 \mathrm{bp}$, which encodes a thiol peroxidase, to 4,809 bp, which encodes a NAD-specific 
Table 1. Genome information for the Glutamicibacter sp. FBE-19 strain sequenced in this study

\begin{tabular}{lc} 
Features & Results \\
Genome size (bp) & $3,846,453$ \\
GC content $(\%)$ & $60.1 \%$ \\
Number of total genes & 3,791 \\
Number of coding genes & 3,704 \\
Number of contigs & $22(>1,000 \mathrm{bp})$ \\
Genome coverage & $181 X$ \\
$N_{50}(\mathrm{bp})$ & 334,508 \\
Maximum contig size (bp) & 798,790 \\
Ribosomal RNA operons & 3 \\
Transfer RNAs & 65 \\
Accession number & JACKQNO00000000 \\
Number of unannotated genes & $1,217(34 \%)$ \\
\hline
\end{tabular}

glutamate dehydrogenase. In addition, the RASTtk annotation identified 65 transfer RNAs and three complete rRNA operons. The genome includes 105 tandem repeats, as identified with the Tandem Repeats Finder (version 4.09) (Benson 1999). Using multilocus sequence typing of the 53 bacterial ribosomal protein subunits (Jolley et al. 2012), we identified strain FBE-19 as G. halophytocola (Fig. 1B).

Multiple genes were identified that may contribute to the antagonist activity of Glutamicibacter FBE-19 against other bacteria. We used antiSMASH (version 5.1.2) to detect gene clusters potentially capable of secondary metabolite synthesis. In particular, FEB-19 has biosynthetic gene clusters predicted to synthesize stenothricin, the $\beta$-lactone salinosporamide $A$, a second unidentified $\beta$-lactone compound, and a carotenoid. The FBE-19 genome is rich in proteins associated with iron scavenging, including the biosynthetic genes for desferrioxamine (also identified by antiSMASH), and several ABC-type siderophore transport systems, including for the siderophore petrobactin. The FBE-19 genome shows relatively little evidence of horizontal gene transfer, having only two clear toxin-antitoxin systems and four transposases. Finally, the FBE-19 genome has multiple features associated with osmoadaptation, including genes encoding an aquaporin and multiple transporters for osmoprotectant compounds, including OpuD for glycine betaine, BetT for choline, and three proline/glycine betaine ProP transporters.

Data availability. Glycerol stocks of Glutamicibacter sp. strain FBE-19 are available for academic purposes upon request. The genome sequence of FBE-19 is available in NCBI under the accession number JACKQN000000000, BioProject: PRJNA655947, and BioSample: SAMN15762367.

\section{Literature Cited}

Andrews, S. 2010. FastQC: A quality control tool for high throughput sequence data. https://www.bioinformatics.babraham.ac.uk/projects/fastqc

Bankevich, A., Nurk, S., Antipov, D., Gurevich, A. A., Dvorkin, M., Kulikov, A. S., Lesin, V. M., Nikolenko, S. I., Pham, S., Prjibelski, A. D., Pyshkin, A. V., Sirotkin, A. V., Vyahhi, N., Tesler, G., Alekseyev, M. A., and Pevzner, P. A. 2012. SPAdes: A new genome assembly algorithm and its applications to single-cell sequencing. J. Comput. Biol. 19:455-477.

Benson, G. 1999. Tandem repeats finder: A program to analyze DNA sequences. Nucleic Acids Res. 27:573-580.

Besemer, J., Lomsadze, A., and Borodovsky, M. 2001. GeneMarkS: A self-training method for prediction of gene starts in microbial genomes. Implications for finding sequence motifs in regulatory regions. Nucleic Acids Res. 29:2607-2618.

Brettin, T., Davis, J. J., Disz, T., Edwards, R. A., Gerdes, S., Olsen, G. J., Olson, R., Overbeek, R., Parrello, B., Pusch, G. D., Shukla, M., Thomason, J. A., III, Stevens, R., Vonstein, V., Wattam, A. R., and Xia, F. 2015. RASTtk: A modular and extensible implementation of the RAST algorithm for building custom annotation pipelines and annotating batches of genomes. Sci. Rep. 5:8365.

Busse, H.-J. 2016. Review of the taxonomy of the genus Arthrobacter, emendation of the genus Arthrobacter sensu lato, proposal to reclassify selected species of the genus Arthrobacter in the novel genera Glutamicibacter gen. nov., Paeniglutamicibacter gen. nov., Pseudoglutamicibacter gen. nov., Paenarthrobacter gen. nov. and Pseudarthrobacter gen. nov., and emended description of Arthrobacter rosea. Int. J. Syst. Evol. Microbiol. 66:9-37.

Feng, W.-W., Wang, T.-T., Bai, J.-L., Ding, P., Xing, K., Jiang, J.-H., Peng, X., and Qin, S. 2017. Glutamicibacter halophytocola sp. nov., an endophytic actinomycete isolated from the roots of a coastal halophyte, Limonium sinense. Int. J. Syst. Evol. Microbiol. 67:1120-1125

Irlinger, F., Bimet, F., Delettre, J., Lefèvre, M., and Grimont, P. A. D. 2005. Arthrobacter bergereisp. nov. and Arthrobacter arilaitensis sp. nov., novel coryneform species isolated from the surfaces of cheeses. Int. J. Syst. Evol. Microbiol. 55:457-462.

Ji, J., Yuan, D., Jin, C., Wang, G., Li, X., and Guan, C. 2020. Enhancement of growth and salt tolerance of rice seedlings (Oryza sativa L.) by regulating ethylene production with a novel halotolerant PGPR strain Glutamicibacter sp. YD01 containing ACC deaminase activity. Acta Physiol. Plant. 42:42.

Jolley, K. A., Bliss, C. M., Bennett, J. S., Bratcher, H. B., Brehony, C., Colles, F. M., Wimalarathna, H., Harrison, O. B., Sheppard, S. K., Cody, A. J., and Maiden, M. C. J. 2012. Ribosomal multilocus sequence typing: Universal characterization of bacteria from domain to strain. Microbiology 158:1005-1015.

Kanehisa, M., Sato, Y., Kawashima, M., Furumichi, M., and Tanabe, M. 2016. KEGG as a reference resource for gene and protein annotation. Nucleic Acids Res. 44: D457-D462.

Mikheenko, A., Prijbelski, A., Saveliev, V., Antipov, D., and Gurevich, A. 2018. Versatile genome assembly evaluation with QUAST-LG. Bioinformatics 34:1142-i150. 
Nishu, S. D., Hyun, H. R., and Lee, T. K. 2019. Complete genome sequence of drought tolerant plant growth-promoting rhizobacterium Glutamicibacter halophytocola DR408. Korean J. Microbiol. 55:300-302.

Qin, S., Feng, W.-W., Zhang, Y.-J., Wang, T.-T., Xiong, Y.-W., and Xing, K. 2018. Diversity of bacterial microbiota of coastal halophyte Limonium sinense and amelioration of salinity stress damage by symbiotic plant growth-promoting actinobacterium Glutamicibacter halophytocola KLBMP 5180. Appl. Environ. Microbiol. 84:e01533-18.

Roberts, D. C., Fleischer, S. J., Sakamoto, J. M., and Rasgon, J. L. 2018. Potential biological control of Erwinia tracheiphila by internal alimentary canal interactions in Acalymma vittatum with Pseudomonas fluorescens. J. Appl. Microbiol. 125: 1137-1146.

Rojas, E. S., Batzer, J. C., Beattie, G. A., Fleischer, S. J., Shapiro, L. R., Williams, M. A., Bessin, R., Bruton, B. D., Boucher, T. J., Jesse, L. C. H., and Gleason, M. L. 2015. Bacterial wilt of cucurbits: Resurrecting a classic pathosystem. Plant Dis. 99: 564-574.
Santos, R. G., Hurtado, R., Gomes, L. G. R., Profeta, R., Rifici, C., Attili, A. R., Spier, S. J., Giuseppe, M., Morais-Rodrigues, F., Gomide, A. C. P., Brenig, B., Gala-García, A., Cuteri, V., Castro, T. L. de P., Ghosh, P., Seyffert, N., and Azevedo, V. 2020. Complete genome analysis of Glutamicibacter creatinolyticus from mare abscess and comparative genomics provide insight of diversity and adaptation for Glutamicibacter. Gene 741:144566.

Shapiro, L. R., Paulson, J. N., Amold, B. J., Scully, E. D., Zhaxybayeva, O., Pierce, N. E., Rocha, J., Klepac-Ceraj, V., Holton, K., and Kolter, R. 2018. An introduced crop plant is driving diversification of the virulent bacterial pathogen Erwinia tracheiphila. MBio 9:e01307-18.

Vesuna, A. P., and Nerurkar, A. S. 2020. Biocontrol impact of AHL degrading actinobacteria on quorum sensing regulated virulence of phytopathogen Pectobacterium carotovorum subsp. carotovorum BR1. Plant Soil 453:371-388.

Xiong, Y.-W., Gong, Y., Li, X.-W., Chen, P., Ju, X.-Y., Zhang, C.-M., Yuan, B., Lv, Z.-P., Xing, K., and Qin, S. 2019. Enhancement of growth and salt tolerance of tomato seedlings by a natural halotolerant actinobacterium Glutamicibacter halophytocola KLBMP 5180 isolated from a coastal halophyte. Plant Soil 445:307-322. 